\title{
Palladium Nanosheets as Safe Radiosensitizers for Radiotherapy
}

Yao-Wen Jiang ${ }^{\dagger}$, Ge Gao ${ }^{\dagger}$, Hao-Ran Jia ${ }^{\dagger}$, Xiaodong Zhang ${ }^{\dagger}$, Xiaotong Cheng ${ }^{\dagger}$, Hong-Yin Wang ${ }^{\dagger}$, Peidang Liu ${ }^{\ddagger}$, and Fu-Gen $\mathrm{Wu}^{\dagger}$,*

${ }^{\dagger}$ State Key Laboratory of Bioelectronics, School of Biological Science and Medical Engineering, Southeast University, Nanjing 210096, P. R. China

Institute of Neurobiology, School of Medicine, Southeast University, Nanjing 210096, P. R. China

Corresponding Author

*E-mail:wufg@seu.edu.cn 


\section{Experimental Section}

Materials. Palladium chloride $\left(\mathrm{PdCl}_{2}\right)$, concentrated hydrochloric acid $(\mathrm{HCl})$, and dimethylformamide (DMF) were purchased from Sigma-Aldrich. Carbon monoxide was ordered from Nanjing Wenda Special Gas Co., Ltd. Thiol poly(ethylene glycol) methoxyl (HS-PEG, M.W. 2 kDa) and thiol poly(ethylene glycol) amine (HS-PEG-MH 2 , M.W. 2 kDa) were obtained from Beijing JenKem Technology Co., Ltd. (China). Fluorescein isothiocyanate (FITC), 3-(4,5-dimethylthiazol-2-yl)-2,5-diphenyltetrazolium bromide (MTT), and dimethyl sulfoxide (DMSO) were provided by J\&K Chemicals (China). 4\% Glutaraldehyde, Triton X-100, ethanol, bovine serum albumin (BSA), and nitric acid (electronic grade) were bought from Aladdin Chemistry Co. Ltd. (China). All solutions were prepared with deionized (DI) water $(18.2 \mathrm{M} \Omega \cdot \mathrm{cm})$ purified by a Milli-Q system (Millipore).

Preparation and Modification of Palladium Nanosheets (Pd NSs). Reaction between $\mathrm{PdCl}_{2}$ and concentrated $\mathrm{HCl}$ at $70{ }^{\circ} \mathrm{C}$ for $1 \mathrm{~h}$ was used for $\mathrm{H}_{2} \mathrm{PdCl}_{4}$ aqueous solution preparation. $10 \mathrm{~mL}$ of DMF containing $60 \mu \mathrm{L}$ of $\mathrm{H}_{2} \mathrm{PdCl}_{4}$ solution $(1 \mathrm{M})$ was placed in a flask followed by the introduction of 1 atm $\mathrm{CO}$ atmosphere. The solution was subject to vigorous stirring until the color of the reaction solution changed from brown red to lemon yellow, indicating the formation of dinuclear $\mathrm{Pd}^{\mathrm{I}}$ carbonyl chloride complex. After the completion of the reaction, $\mathrm{CO}$ gas was removed and then $2 \mathrm{~mL}$ of $\mathrm{DI} \mathrm{H}_{2} \mathrm{O}$ was injected into the flask under stirring. A fast color change from light yellow to dark blue can be seen and this reaction was allowed to proceed for $10 \mathrm{~min}$ for completion. Then excess HS-PEG was added immediately to the above dispersion containing the formed Pd NSs. After reaction for $3 \mathrm{~h}$ at room temperature, the obtained dispersion was dialyzed against water to remove 
unreacted HS-PEG. Finally, the PEGylated Pd NS dispersion was stored at $\sim 4{ }^{\circ} \mathrm{C}$ for further use. Besides, FITC was chosen to label the Pd NSs for endocytosis tracking. Briefly, HS-PEG and HS-PEG-FITC were mixed in a molar ratio of 98:2 and introduced into the freshly prepared Pd NS dispersion with vigorous stirring for $3 \mathrm{~h}$ at room temperature (HS-PEG-FITC was prepared by the addition reaction between FITC and $\mathrm{HS}-\mathrm{PEG}-\mathrm{NH}_{2}$ ). Excessive PEG derivatives were removed by dialysis against water to obtain FITC-labeled Pd NSs (denoted as Pd NSs-FITC).

Characterization of Pd NSs. The size and morphology of Pd NSs were characterized using a transmission electron microscope (TEM) (JEOL JEM-2100, Japan), and the size distribution histogram of Pd NSs was obtained from the representative TEM images with Nano Measurer software (Version 1.2). UV-vis spectrum was collected on a UV-vis spectrophotometer (UV-2600, Shimadzu, Japan). The concentration of the prepared Pd NS dispersion was determined with inductively coupled plasma optical emission spectrometry (ICP-OES) (Optima 5300DV, PerkinElmer, USA) using the standard stock solution of Pd element (National Research Center for Certified Reference Materials, China), and the obtained mass concentration of Pd element was used for the experiments mentioned below.

Cell Culture and Cellular Uptake of Pd NSs. Human breast cancer cells (MCF-7) were cultured in complete Dulbecco's modified Eagle's medium (cDMEM) (containing DMEM, $10 \%$ fetal bovine serum (FBS), $100 \mathrm{U} / \mathrm{mL}$ penicillin, and $100 \mu \mathrm{g} / \mathrm{mL}$ streptomycin) in an incubator with $5 \% \mathrm{CO}_{2}$ at $37{ }^{\circ} \mathrm{C}$. Flow cytometric experiments of MCF-7 cells interacting with different concentrations of Pd NSs $(0,10,30$, and $60 \mu \mathrm{g} / \mathrm{mL}$, based on Pd element) were performed on a flow cytometer (NovoCyte 2070R, ACEA Biosciences, Inc., USA) using 
NovoExpress 1.0 software. For the quantitative analysis of cellular nanoparticles, the cells were washed with phosphate-buffered saline (PBS) and harvested. Then the mean intensity of side-scattered (SSC) light from Pd NSs in the cells was measured via flow cytometry. Apart from that, confocal laser scanning microscopy was used to observe the MCF-7 cells treated with different concentrations of Pd NSs-FITC $(0,10,30$, and $60 \mu \mathrm{g} / \mathrm{mL}$, based on Pd element). Nuclei were stained blue with Hoechst 33342 (Beyotime, China). The locations of lysosomes were marked by Lyso-Tracker Red (Invitrogen, USA).

Cytotoxicity Evaluation. MCF-7 cells were seeded in a 96-well plate with a density of $5 \times$ $10^{3}$ cells/well. After $24 \mathrm{~h}$ culture, cells were exposed to different concentrations of Pd NSs (0, $3,10,30,60$, and $100 \mu \mathrm{g} / \mathrm{mL}$, based on Pd element) in cDMEM for another $24 \mathrm{~h}$. Then the culture medium in each well was replaced by $100 \mu \mathrm{L}$ of $0.5 \mathrm{mg} / \mathrm{mL}$ MTT in cDMEM. After 5 $\mathrm{h}$ of incubation, the MTT solution was removed and $150 \mu \mathrm{L}$ DMSO was added to each well. After shaking for $10 \mathrm{~min}$, the absorbance at $492 \mathrm{~nm}$ of each well was measured using a microplate photometer (Multiskan FC, Thermo).

Hemolysis Assay. To assess the hemocompatibility of Pd NSs, clean red blood cells (RBCs) were obtained by removing serum from the blood of a healthy mouse and dispersed in PBS at a density of $2 \times 10^{8}$ cells $/ \mathrm{mL}$. Then, $150 \mu \mathrm{L}$ of RBC suspension was mixed separately with $150 \mu \mathrm{L}$ of $\mathrm{Pd}$ NS dispersion with a final Pd concentration of 5, 10, 20, 30, 60, 80, and 100 $\mu \mathrm{g} / \mathrm{mL}$ (Pd element), respectively, followed by incubation at $37^{\circ} \mathrm{C}$ for $2 \mathrm{~h}$ (negative control: RBCs in PBS; positive control: RBCs in 1\% Triton X-100). Finally, after removing the supernatants of the centrifuged RBC suspensions that experienced different treatments, an equal amount of $1 \%$ Triton $\mathrm{X}-100$ was introduced into each precipitate, followed by the 
measurement of the absorbance at $450 \mathrm{~nm}(I)$ to quantify the remaining hemoglobin. The hemolysis rate was calculated according to the following formula:

Hemolysis $(\%)=\left(I_{\text {negative control }}-I_{\text {sample }}\right) /\left(I_{\text {negative control }}-I_{\text {positive control }}\right) \times 100 \%$ Apoptosis/Necrosis Analysis. An annexin V-FITC/propidium iodide (PI) apoptosis detection kit (KeyGen Biotech., China) was used for evaluating cell apoptosis/necrosis rates. Briefly, MCF-7 cells $\left(5 \times 10^{5}\right.$ cells) were exposed to different concentrations of Pd NSs for $24 \mathrm{~h}$, and then the cells were washed twice with PBS solution and harvested with trypsin solution. Afterwards, the cells were incubated with $500 \mu \mathrm{L}$ binding buffer containing $5 \mu \mathrm{L}$ annexin V-FITC solution and $5 \mu \mathrm{L}$ PI solution for $15 \mathrm{~min}$ at room temperature in the dark. Finally, flow cytometric experiments were performed to analyze the cell apoptosis/necrosis rates. The apoptosis/necrosis analyses for cells after X-ray irradiation were the same as mentioned above.

Colony Formation Assay. MCF-7 cells were cultured in 24 -well plates $\left(5 \times 10^{4}\right.$ cells per well) for $24 \mathrm{~h}$. Cells treated without (control) or with Pd NSs $(60 \mu \mathrm{g} / \mathrm{mL}$, based on Pd element) for $24 \mathrm{~h}$ were irradiated by X-rays generated by a linear accelerator at various doses of $0,2,4,6$, and $8 \mathrm{~Gy}$, respectively. Next, cells were washed and trypsinized, and then 1000 cells were seeded in each well of 6-well plates with $2 \mathrm{~mL}$ of fresh culture medium. Cells in plates were allowed to grow for 7 days followed by Giemsa staining using a Giemsa's solution (KeyGen Biotech., China). The colonies formed with more than 50 cells were counted. Cell survival fraction and sensitization enhancement ratio (SER) were determined by a classical multitarget single-hit model $\left(\mathrm{S}=1-\left(1-\mathrm{e}^{-D / D 0}\right)^{n}\right)$, where $\mathrm{S}$ is the survival fraction, $D$ is the radiation dose, $D_{0}$ is the mean lethal dose, and $n$ is the extrapolation 
number. ${ }^{1}$ The final survival fraction curve of each group was generated via a nonlinear fitting using Origin 8.0, and the SER was expressed with the following formulas: SER $=D_{\mathrm{q}}$ (control group) $/ D_{\mathrm{q}}$ (treated group) and $D_{\mathrm{q}}=\ln (n) \times D_{0}$, where $D_{\mathrm{q}}$ is the quasi-threshold dose.

Real-Time Cellular Analysis (RTCA). For RTCA experiments, the trypsinized MCF-7 cells were counted and seeded on an electronic 8 -well plate (with $1.5 \times 10^{3}$ cells in $500 \mu \mathrm{L}$ cDMEM in each well) of RTCA iCELLigence analyzer (ACEA Biosciences, Inc.) for cell proliferation and growth assays. The RTCA instrument was placed in the incubator $\left(37^{\circ} \mathrm{C}\right.$, $\left.5 \% \mathrm{CO}_{2}\right)$ and the data were transmitted to an iPad via wireless Internet. The software in the iPad friendly allowed for real-time interfacing with the electronic 8-well plate and included real-time data display and analysis functions.

ROS Generation Measurement. To measure the generation of intracellular ROS, trypsinized MCF-7 cells were loaded with $10 \mu \mathrm{M}$ dichlorodihydrofluorescein diacetate (DCFH-DA, KeyGen Biotech., China) in DMEM without serum for $20 \mathrm{~min}$ in the dark (37 ${ }^{\circ} \mathrm{C}, 5 \% \mathrm{CO}_{2}$ ) and were mixed per 4 min to keep the samples homogeneous. Next, cells were washed with PBS for 3 times to remove excess DCFH-DA and finally resuspended in PBS. Flow cytometric analysis was immediately carried out for the above cells. For each sample, the mean fluorescence intensity (MFI) of 20,000 cells was recorded to quantify the intracellular ROS.

Cell Cycle Distribution Assay. Cell cycle distribution was assayed using a cell cycle detection kit (KeyGen Biotech., China). In brief, treated MCF-7 cells were trypsinized and washed with PBS, and then fixed with precooled $70 \%$ ethanol at $4{ }^{\circ} \mathrm{C}$. Prior to staining, the $70 \%$ ethanol was removed followed by PBS washing. Cells were incubated with $100 \mu \mathrm{L}$ of 
RNase A at $37{ }^{\circ} \mathrm{C}$ for $30 \mathrm{~min}$. Then $400 \mu \mathrm{L}$ of $50 \mu \mathrm{g} / \mathrm{mL}$ PI solution was added and co-incubated with cells in the dark for 30 min at $4{ }^{\circ} \mathrm{C}$. Finally, the cell cycle distribution was analyzed by flow cytometry. For each sample, 20,000 cells were recorded for analysis.

Detection of DNA Double-Stranded Breaks. Phosphorylated histone H2AX has been widely regarded as a molecular marker for DNA double-stranded breaks. Due to the correlation between the amount of phosphorylated histone $\mathrm{H} 2 \mathrm{AX}$ and the degree of DNA damage, specific immunofluorescence assay was performed to detect phosphorylated histone $\mathrm{H} 2 \mathrm{AX}$ at Ser $139(\gamma-\mathrm{H} 2 \mathrm{AX})$. Briefly, after Pd NS and/or X-ray treatment, the cells were washed twice with PBS, fixed with $4 \%$ glutaraldehyde for $10 \mathrm{~min}$, and permeated with $1 \%$ Triton X-100. Next, the cells were blocked in $1 \%$ BSA for $1 \mathrm{~h}$ to avoid nonspecific protein adsorption and then incubated with Alexa Fluor 488-labeled anti- $\gamma-\mathrm{H} 2 \mathrm{AX}$ antibody (eBioscience, USA) overnight at $4{ }^{\circ} \mathrm{C}$. Nuclei were stained blue with Hoechst 33342.

In Vivo Experiments. All animal experimental procedures were performed under protocols approved by the Animal Care Committee of Southeast University and conducted in compliance with the Regulations for the Administration of Affairs Concerning Experimental Animals of China. The mouse cervical cancer cells (U14 cells) $\left(1 \times 10^{6}\right)$ suspended in $100 \mu \mathrm{L}$ PBS were subcutaneously implanted into the back of each BALB/c nude mouse to generate the U14 xenograft tumor model. The mice were used for further experiments when the tumor reached approximately $90 \mathrm{~mm}^{3}$ in volume. For the in vivo tumor growth study, the mice intratumorally injected with PBS or Pd NS (10 mg/kg, based on Pd element) dispersion were exposed to different doses of X-ray ( 0 or 6 Gy). After the above treatments, the tumor volume and mouse weight were measured daily until day 12 . The tumor volume was calculated by 
(length of tumor $) \times(\text { width of tumor })^{2} / 2$. The relative tumor volume was calculated by $V / V_{0}$, where $V_{0}$ is the initial tumor volume, and $V$ is the volume of the tumor measured at different time points. Finally, all mice were sacrificed and the tumor tissues were separated for taking photos and weighing.

Ex Vivo Histological Staining. To investigate the systemic toxicity of Pd NSs, representative mice were sacrificed on the 12th day after treatments and the major organs including hearts, livers, spleens, lungs, and kidneys were dissected and then fixed in $4 \%$ formaldehyde solutions, followed by making paraffin sections. Next, the paraffin sections were stained with hematoxylin and eosin (H\&E) following the standard protocol. All sections were observed on a Leica TCS SP8 microscope.

Hemanalysis. Blood samples were collected from the healthy nude mice without treatment or the nude mice with Pd NS treatment for 12 days. The changes of the numbers of red blood cells (RBC) and white blood cells (WBC), the concentration of hemoglobin (HGB), mean corpuscular volume $(\mathrm{MCV})$, mean corpuscular hemoglobin $(\mathrm{MCH})$, mean corpuscular hemoglobin concentration (MCHC), and platelet (PLT) count were analyzed using an automatic hematology analyzer (BC-2800Vet, Mindray, China).

Biodistribution of Pd Element. The organs of Pd NS-treated mice were weighed and digested in highly pure nitric acid and the concentration of $\mathrm{Pd}$ in the digestion solution was measured using ICP-OES.

Statistical Analysis. All obtained data were repeated three times and presented in the form of mean \pm standard deviation (mean $\pm \mathrm{SD}$ ). The results were subjected to one-way analysis of variance (ANOVA) using Tukey's test to analyze the statistical significance between the 
control group and the Pd NS- and/or X-ray-treated groups with the following $p$ values: (ns) for nonsignificant, $\left(^{*}\right)$ for $p<0.05,(* *)$ for $p<0.01$, and $(* * *)$ for $p<0.001$.

\section{Additional Figures}

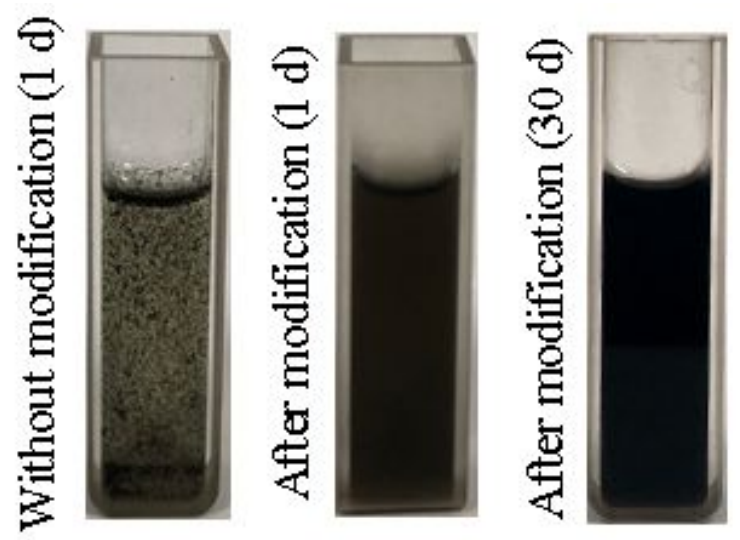

Figure S1. Photograph of Pd NS dispersions $(0.1 \mathrm{mg} / \mathrm{mL}$, based on Pd element $)$ without/with PEG modification after storage for different time periods.

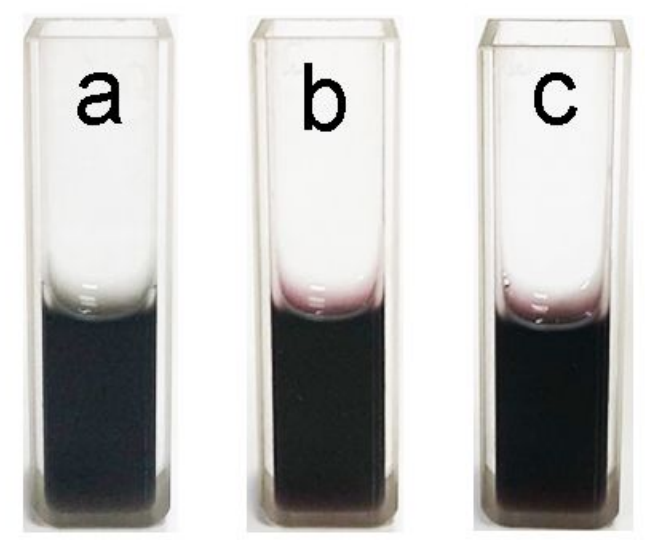

Figure S2. Photograph of Pd NS dispersions $(0.1 \mathrm{mg} / \mathrm{mL}$, based on Pd element) in (a) PBS, (b) DMEM, and (c) cDMEM (containing $10 \%$ FBS). 


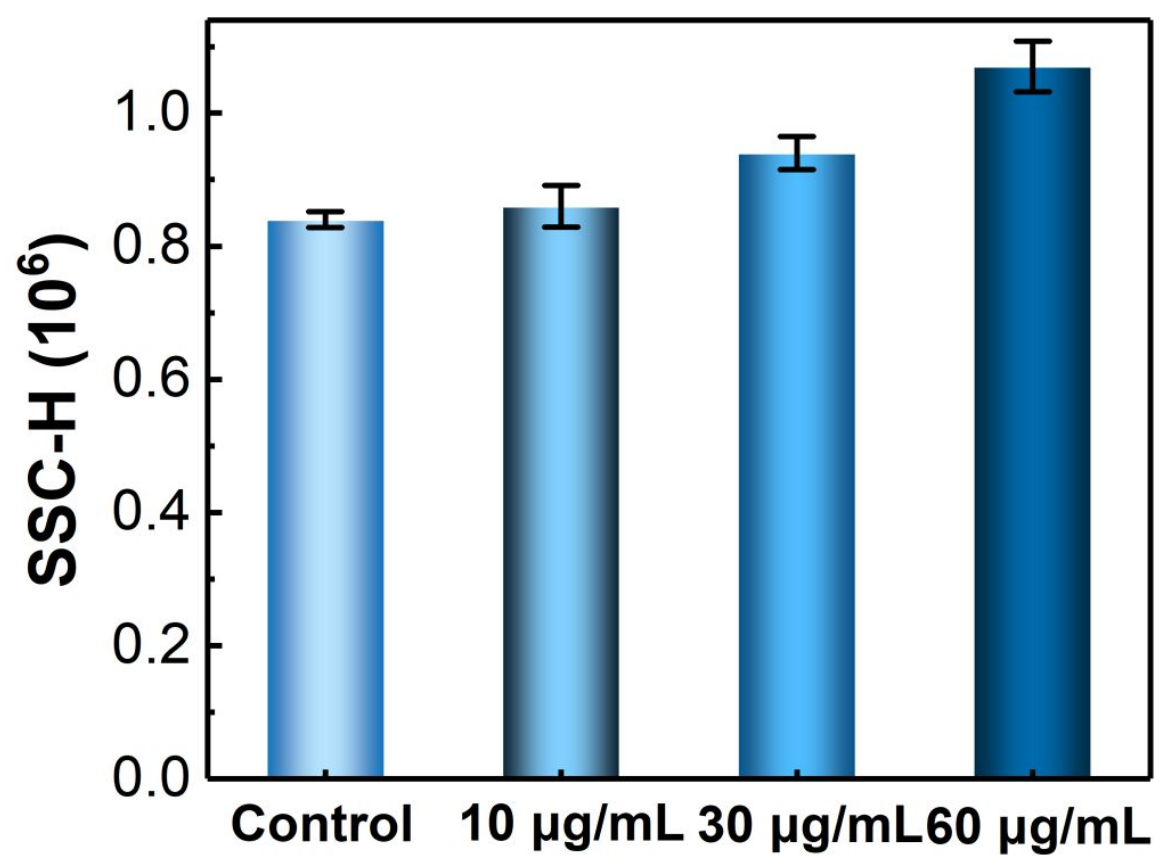

Figure S3. Quantitative analysis results of Figure 4a.

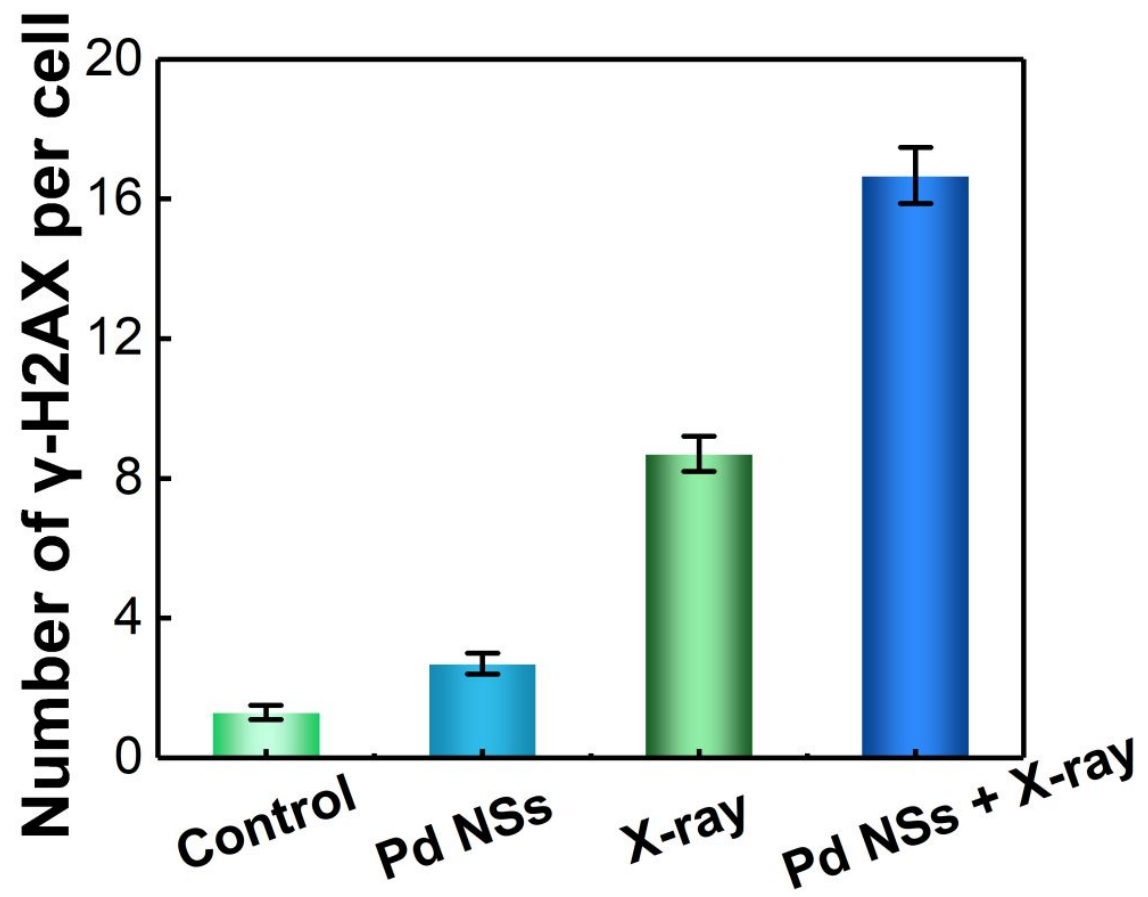

Figure S4. Quantitative analysis results of DNA double-stranded breaks measured via the expression of $\gamma-\mathrm{H} 2 \mathrm{AX}$. 


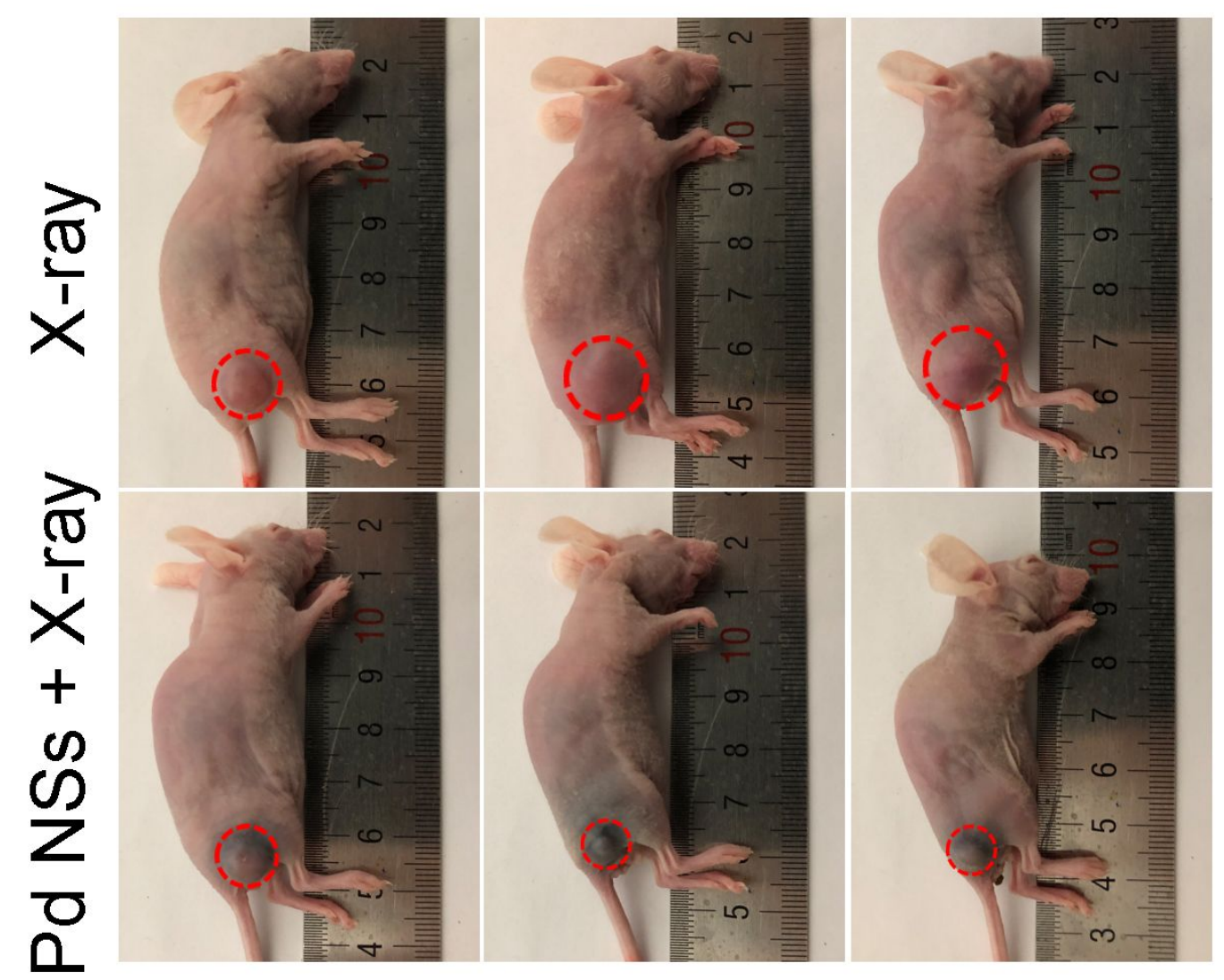

Figure S5. Photographs of U14 tumor-bearing nude mice taken on the 12th day after X-ray treatment or Pd NSs + X-ray treatment.

\section{REFERENCE}

(1) Zhang, X. D.; Wu, D.; Shen, X.; Chen, J.; Sun, Y. M.; Liu, P. X.; Liang, X. J. Size-Dependent Radiosensitization of PEG-Coated Gold Nanoparticles for Cancer Radiation Therapy. Biomaterials 2012, 33, 6408-6419. 